\title{
A Review of Telephone Urological Follow-Up in a District General Hospital During the COVID-19 Pandemic
}

\author{
Daniel Wignall $^{1}$ (1) $\cdot$ Ling Lee $^{1} \cdot$ Agapios Gkentzis $^{1}$
}

Accepted: 29 October 2020 / Published online: 9 November 2020

(C) Springer Nature Switzerland AG 2020

\begin{abstract}
COVID-19 has had an unprecedented effect on healthcare with certain services being altered to minimise face-to-face contact. This aim of this project was to explore opinions on routine telephone consultations and whether they could be implemented as COVID pressures change. We randomly selected 100 patients who had a telephone consultation instead of their normal urological follow-up and posted them a questionnaire. We also compared the number of consultations and non-attendances with the preCOVID era. Seventy percent of patients replied to the questionnaire, and $79 \%$ wanted to continue with telephone appointments. Eighty-two percent felt that despite COVID-19, their urological care was unaffected. Generally, the reason that telephone appointments were unacceptable for patients was a preference for face-to-face interaction. The majority expressed a lack of enthusiasm for potential video consultations with only $43 \%$ interested in this. Over $95 \%$ of patients who required investigations still had these tests performed. There was a reduction in patients who 'did not attend (DNAs)' from 7.5\% prior to COVID-19 to $3 \%$ during the peak of the pandemic. This study has demonstrated our patients' satisfaction and acceptance of remote consultation and its efficiency in reducing missed appointments during the pandemic. It is now an option our patients can now choose as their mode of follow-up.
\end{abstract}

Keywords COVID · Outpatient · Telephone $\cdot$ Clinic $\cdot$ Urology

\section{Introduction}

On the 23rd of March 2020, as the COVID-19 pandemic began to put increasing pressure on healthcare services, outpatient appointments were modified to telephone consultations. New referrals and those who required reexamination were seen in person, but the majority of patients were followed up remotely. The telephone appointments aimed to reduce the risk of transmission of coronavirus during the outbreak. On average, there are over twenty patients reviewed on an outpatient clinic list, and

This article is part of the Topical Collection on Covid-19

Daniel Wignall

danwignall@doctors.org.uk

Ling Lee

Ling.lee@boltonft.nhs.uk

Agapios Gkentzis

agapios.gkentzis@boltonft.nhs.uk

1 Bolton Hospital, Bolton, UK the patients are often elderly with co-morbidities. The risk reduction protected staff and patients by reducing footfall in the department.

Naturally there are concerns about widespread integration of telephone consultations due to some of its limitations. Visual cues from the patient can be as useful as what is being said, and this is lost when communicating over the phone. As the clinician conducting the consultation, it can be harder to transmit empathy from loss of these cues which is particularly pertinent when breaking bad news. During the pandemic, it has on occasion been necessary to give upsetting information over the phone, but it is questionable how appropriate this is going forwards. A study done in the primary care setting promotes telephone clinics as a good alternative for following-up patients but expresses a concern surrounding missing pertinent information when still trying to form a diagnosis [1].

Another challenge clinicians face whilst undergoing telephone clinics is overcoming language barriers. The use of an interpreter on loudspeaker is possible, but it is difficult for the clinician to be sure that the correct message has been conveyed and may break confidentiality. It is a similar principle with patients who are hard 
Fig. 1 Bar chart showing feedback on telephone appointments
Feedback on telephone consultations

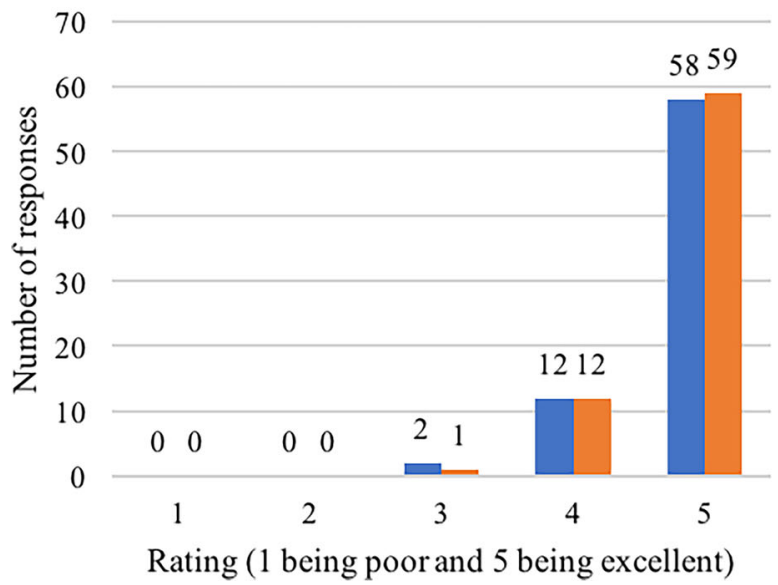

- How did you find your recent telephone appointment?

aid you feel listened to and was your problem addressed today? of hearing with sign language interpretation. These patients often present with a family member or carer who can mediate, but a conversation over the phone is a much more exclusive form of communication.

Video calls have been trialled within our hospital; however, these are yet to be widely rolled out. Studies looking at the use of telemedicine have shown it to be an acceptable alternative by patients and many expressing a preference for remote follow-up [2].

The aim of this project was to assess whether the follow-up telephone appointments were an acceptable, appropriate, and adequate way of following-up urology patients and whether they should continue as COVID-19 restrictions are lifted.

\section{Participants and Setting}

In the month of April 2020, a two-sided questionnaire was mailed to 100 patients within a week of their urology follow-up telephone appointment (see Appendix Fig. 6).
This format was felt to be the most appropriate as it allowed patients who lacked computer skills to respond and hopefully allowed patients the freedom to answer questions more honestly than if they were asked over the phone. The replies were returned to us in stamped addressed envelopes.

The first section of the survey was demographic information including age, gender, and the requirement for an interpreter. This was then followed by 9 questions. The first two questions assessed patient's general satisfaction and whether they felt their problem was adequately dealt with over the telephone. This was scored from one (poor) to five (excellent) to assess fitness for purpose.

The next two explored the difficulties patients had with attending the hospital for appointments and challenges they experienced with telephone appointments. They could select from a multiple choice list of common obstacles.

There were then four 'yes or no' questions which were:

- In the future would you ever want a video call?
Fig. 2 Bar chart to show difficulties patient have in attending hospital appointments

\section{Do any of the following make it difficult for you to attend hospital appointments?}

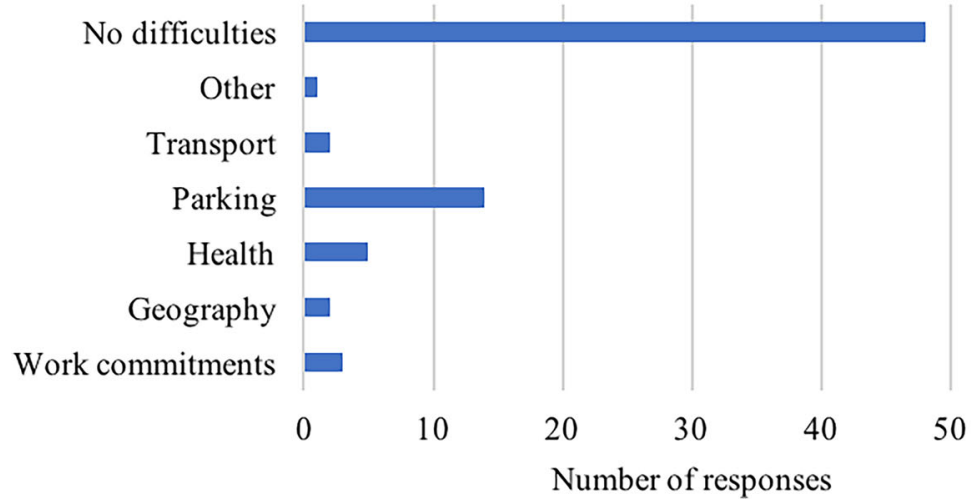

SN Comprehensive Clinical Medicine A SPRINGER NATURE journal 
Fig. 3 Bar chart to show difficulties patients have using telephone appointments
Would any of the following stop you using telephone appointments?

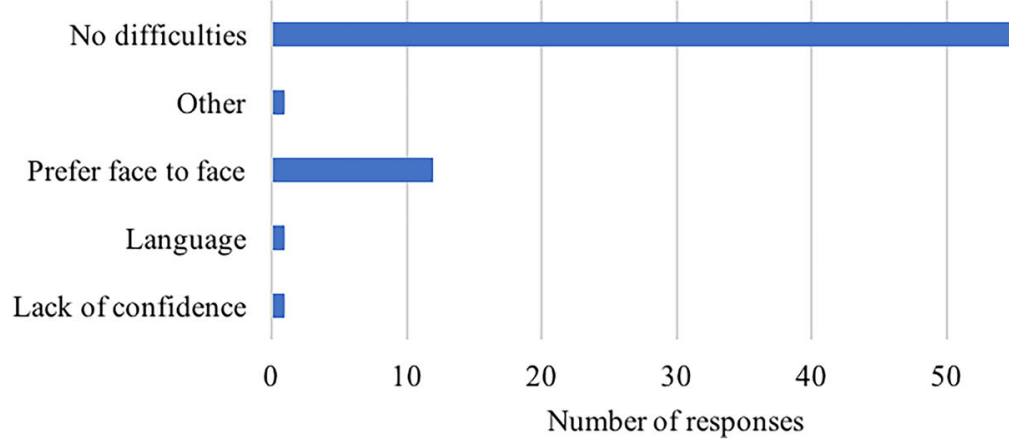

- Were you expected to have any tests/investigations before your appointment?

- If yes, did the telephone appointment stop you getting these tests?

- Would you like your next appointment to be via the telephone?

The final question was whether the patient felt that COVID19 has had a negative impact on their urological treatment. This was a scale from 1 (negative impact) to 10 (positive impact).

Alongside this survey, a record of missed appointments was kept for 7 weeks from a single consultant's clinic during the peak of COVID-19. This was compared with 7 weeks from December before the implementation of telephone appointments for follow-up.

\section{Results}

We received 70 responses to our postal questionnaire with 59 males and 11 females. The median age of the patient who received our survey was 73 years old with a wide age range of 24 to 93 . Only one patient noted a requirement for an interpreter.

There was a very positive response for the outcomes in the telephone follow-up clinic. When asked how they regarded the telephone appointment, over 95\% score it four or five (on a scale of one to five with five being excellent). Similarly, over $95 \%$ of patients felt listened to and that their problem was adequately addressed over the telephone (see Fig. 1).

The follow-up to this addressed any specific points which might deter people from engaging with telephone follow-up and any features that could allow flexibility patients. When
Fig. 4 Pie chart to show whether remote clinic points will deter patients from getting their investigations

\section{Were you expected to get investigations prior to your clinic appointment?}

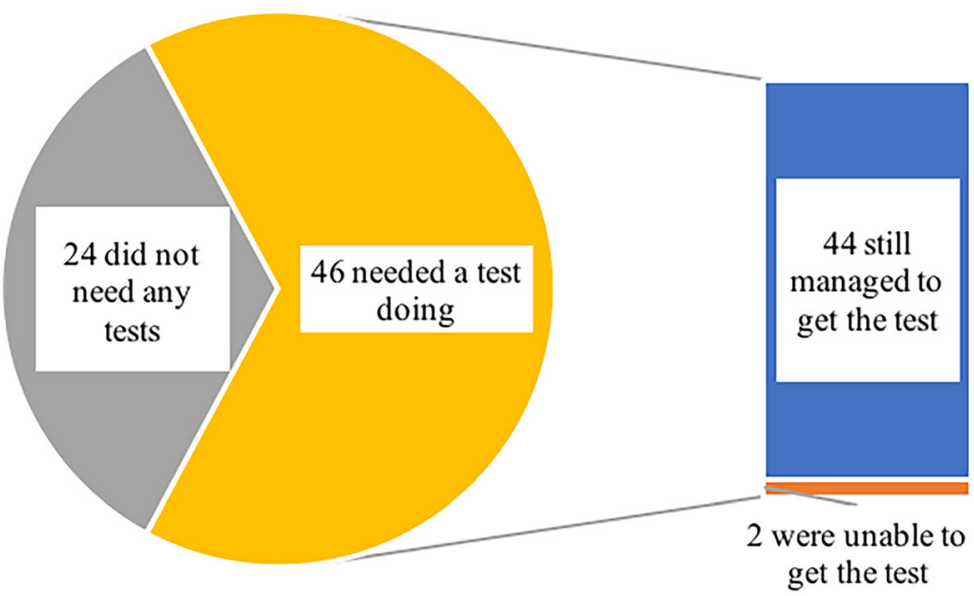


Table 1 Difference in opinion regarding video follow-up divided into age categories

\begin{tabular}{lll}
\hline Age group & Total number & Amenable to video follow-up (\%) \\
\hline $20-39$ & 2 & $50 \%$ \\
$40-59$ & 6 & $66 \%$ \\
$60-79$ & 49 & $41 \%$ \\
$80+$ & 13 & $38 \%$ \\
\hline
\end{tabular}

asked if the patients had any difficulty in attending the hospital, 48/70 reported they had none. The most common problem people have when attending hospital appointments was parking; however, there were also difficulties with work commitments and health problems (see Fig. 2). The results suggest that the main reason people would not want to continue with telephone appointments is a preference for face-to-face appointments (see Fig. 3). This strongly correlated with the response to the final question on whether the patient would be happy with a telephone appointment next time.

The results of this section were reassuring with only two patients $(<5 \%)$ stating that the new system was a barrier to them undergoing the investigations. Figure 4 demonstrates this.

Regarding future video consultations, $57 \%$ of patients would choose not to have follow-up in this manner. Table 1 demonstrates patient opinion on video calls and their age. The least positive about the idea were the respondents over the age of 80 . Of note, there were only two patients within the $20-40$ age group.

Seventy-nine percent of patients wish to continue with telephone consultations as part of their long-term follow-up. One hundred percent of female patients wish to continue with telephone appointments and $76 \%$ of men. The table below demonstrates the split of opinion by age (see Table 2).

When comparing the pre-COVID-19 outpatient sessions to the COVID-19 sessions, there was a decrease in missed appointments by $4.5 \%$. Prior to the virus outbreak, on average $7.5 \%$ of follow-up patients did not attend which was reduced to $3 \%$ of patients not contactable by telephone during the pandemic.

Table 2 Difference in opinion regarding telephone follow-up divided into age categories

\begin{tabular}{lll}
\hline Age group & Total number & Amenable to telephone follow-up (\%) \\
\hline $20-39$ & 2 & $100 \%$ \\
$40-59$ & 6 & $83 \%$ \\
$60-79$ & 49 & $80 \%$ \\
$80+$ & 13 & $77 \%$ \\
\hline
\end{tabular}

As a final point, we asked patients whether they felt that the COVID-19 outbreak had negatively affected their urological treatment. The graph below shows the spread of responses from the visual analogue scale (see Fig. 5). The spread of answers was fairly even with some patients at the extremes having had poor/excellent experiences, but the majority of people feeling a normal standard were maintained.

\section{Conclusions}

Our survey showed that over $95 \%$ of patients responded very positively to the telephone follow-up and 79\% asked for their next appointment to continue this way. Another paper published during the COVID outbreak was similar at 83\% [3]. The findings suggest it is appropriate to integrate this format of outpatient clinic for patients who are amenable to it. It can be reviewed at the end of the appointment if the patient is happy to proceed with future telephone appointments. This is part of empowering patients to be involved in their own decision-making.

It was important to hear what challenges people experienced as part of their ongoing follow-up. The wide age range of urological patients means that there are different barriers for different groups. Work commitments in the under 70s cohort and disabling health issues in comorbid elderly patients can make follow-up less straightforward. These are also issues which can potentially be sidestepped by offering more remote clinics. This will allow frail patients to engage with the service from home and cause minimal scheduling disturbances for working patients.

This being said, around two-thirds of our patients were still required to access some form of physical health service to get investigations done. This was hypothesised potentially problematic with remote clinics because patients may not get investigations done when they were not attending the hospital that day. An example of this is a test such as X-ray kidney, ureters, and bladder (KUB) for patients under stone surveillance. It was very reassuring to see that over $95 \%$ of the required investigations were still done prior to the clinic appointment. As a way of reducing the risk of infectious transmission, this still worked as it reduced the amount of time spent in the hospital and the number of staff interactions. For many patients, the investigations could also be done in the primary care setting forgoing the need for any trip into the hospital.

It is reassuring to see the majority of people felt their ongoing care was maintained at a high standard throughout the pandemic and most felt their health did not suffer as a result of this. Continuing high volume clinics through the use of phone calls was possibly a contributor to this satisfaction as it allowed the department to honour previously organised appointments and avoid rescheduling/delaying patients. Anecdotally, patients were very thankful for continued contact 
Fig. 5 Bar chart showing the impact that COVID-19 had on patient's urological care
Do you feek COVID-19 had had an impact on your urological treatment

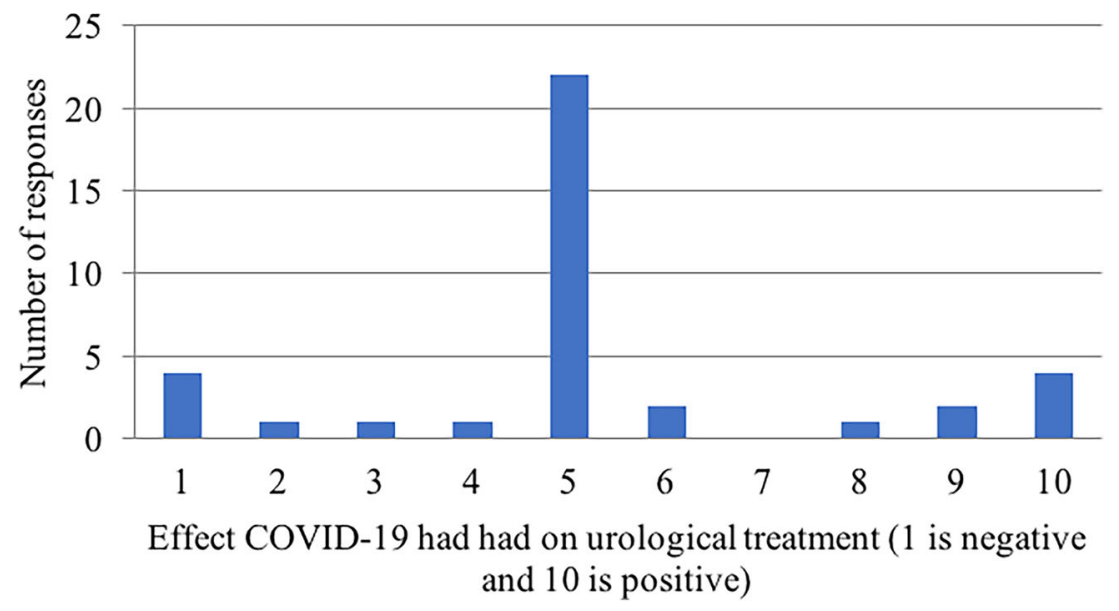

during the pandemic as there is often a lot of anxiety, particularly in patients who are under cancer surveillance. This does bring to light the issue of breaking bad news over the phone and whether this is an appropriate way to communicate upsetting news. During the pandemic, it was an unfortunate necessity, but moving forwards, clinicians should be mindful of whether it would be more appropriate to organise physical appointments. It can be easy to forget the impact of seeing the patient in person to ensure clear and empathetic lines of communication.

There was a less positive response to video consultations with the majority of patients expressing that they would not be interested should that resource be widely rolled out in the future. This is in contrast to other studies which show patient groups responding positively to the integration of video consultations (a). There may be unfamiliarity with the technology as when the responses were sorted into age, the least receptive to the idea were the patients over 60 . With the average age of the patients in this study being 73 , it sheds some light on why the overall verdict was only $43 \%$ of people considering this an acceptable form of review. The number of patients asked who were aged between 20 and 40 was two, so the $50 \%$ response is likely nonrepresentative of the cohort which have been raised with this technology, and a larger study into this cohort would need to be done.

It is no secret that funding in the NHS is stretched, and missed appointments contribute to a proportion of wasted spending. In one clinic, there were 95 patients followed up from December and 93 patients from April/May, so the number of patients scheduled was the same which reflects no loss of efficiency. The number of patients not attending their face-to-face appointments in the months leading up to the start of the outbreak was $7.5 \%$ which is almost one in ten patients. As a contrast, only $3 \%$ of patients were uncontactable during the peak of COVID-19. From our experience, many patients who were spoken to had forgotten they had an appointment, and these are the people that telephone consultations can capitalise on. Hopefully this will save the department money and make most effective use of clinician's time. There is a risk that over time, patients may begin to devalue their clinic appointments forgetting they still require clinician's time, and this may be reflected in a rise of missed appointments. As a department, the aim is to continue through the COVID pandemic with follow-up telephone consultations unless clinically indicated or a patient expresses a strong desire otherwise. When we begin to see a decline in positive COVID-19 cases, there will need to be decisions made regarding which patients return to face-to-face appointments. What will be most telling is when restrictions are lifted and there is reduced risk in visiting the hospital, how many patients will still prefer remote appointments. On the whole, patients have adapted well to the new format of outpatient follow-up; however, in the post-COVID landscape, we should not forget that this is not a 'one size fits all'.

Authors' Contributions Mr. Wignall - Design, data collection, data analysis, write-up

Miss Lee - Design, write-up

Mr. Gkentzis - Feedback on design, write-up

Data Availability Available upon request.

\section{Compliance with Ethical Standards}

Conflict of Interest The authors declare that they have no conflict of interest.

Ethics Approval Not applicable.

Consent to Participate Implied consent through return postage of questionnaires. 


\section{Appendix}

\section{Feedback on urology telephone consultations}

Personal questions: (please leave blank if you would prefer not to say)

Age

Gender.

Ethnicity

Do you require an interpreter? (please circle one) Yes
No

\section{WHS}

Bolton

\section{NHS Foundation Trust}

Dear patient. You recently had a telephone consultation with the urology department. We would like to know what you thought about this. We would greatly appreciate it if you could complete this short survey and return it to us in the stamped addressed envelope provided.

How did you find your recent telephone appointment? (Please circle a number)

(Terrible) 1

2

3

4

5 (Excellent)

(It was neither good nor bad)

Did you feel listened to and were your problems addressed over the phone? (Please circle a number)

$\begin{array}{lllllll}\text { (No) } & 1 & 2 & 3 & 4 & 5 & \text { (Yes) }\end{array}$

Do any of the following make it difficult for you to attend hospital appointments? (Please tick any that apply)

D Geographical location

口 Health problems

․ Social/work commitments

ㄴ Lack of transport

- Role as a carer

․ Hospital parking

ㅁ No difficulties

口 Other

Would any of the following stop you using telephone appointments? (Please tick any that apply)

D Lack of confidence with technology

D You would like to see a doctor face-to-face

口 Language barrier/Lack of interpreter

口 No difficulties

口 Other

Fig. 6 Feedback form sent to the patient 
In the future, would you ever want a video call? (please circle)

Yes

No

Were you expected to have any tests before your appointment? (blood tests, scans or urine tests)

Yes

No

If you answered yes above, did having a telephone appointment stop you getting this test done? (please circle)

Yes

No

Do you feel that COVID-19 has had any impact on your urological treatment? (Please circle a number)

$\begin{array}{llllllllllll}\text { (Negative impact) } & 1 & 2 & 3 & 4 & 5 & 6 & 7 & 8 & 9 & 10 & \text { (Positive impact) }\end{array}$

(No impact)

Would you like your next urology appointment to be via a telephone consultation? (please circle)

Yes

No

Thank-you completing this. Comments?

Fig. 6 (continued)

\section{References}

1. McKinstry B, Hammersley V, Burton C, Pinnock H, Elton R, Dowell $\mathrm{J}$, et al. The quality, safety and content of telephone and face-to-face consultations: a comparative study. Qual Saf Health Care. 2010;19(4):298-303 Available at: https://pubmed.ncbi.nlm. nih.gov/20430933/. Accessed 30 Oct 2020

2. Boehm K, Ziewers S, Brandt MP, Sparwasser P, Haack M, Willems $\mathrm{F}$, et al. Telemedicine online visits in urology during the COVID-19 pandemic-potential, risk factors, and patients' perspective. Eur Urol.
2020;78(1):16-20 Available at: https://pubmed.ncbi.nlm.nih.gov/ 32362498/. Accessed 30 Oct 2020

3. Patel S, Douglas-Moore J. A reflection on an adapted approach from face-to-face to telephone consultations in our urology outpatient department during the COVID-19 pandemic - a pathway for change to future practice? [published online ahead of print. 2020 May 29]. Available at: https://bjui-journals.onlinelibrary.wiley.com/doi/full/ 10.1111/bju.15119. Accessed 30 Oct 2020

Publisher's Note Springer Nature remains neutral with regard to jurisdictional claims in published maps and institutional affiliations. 